\title{
MESURES INVARIANTES SUR LES HYPERGROUPES BY
}

\author{
R. SPECTOR
}

\begin{abstract}
The notion of an invariant or relatively invariant measure on a hypergroup is studied. The main result is that every commutative hypergroup carries an invariant measure.
\end{abstract}

Les hypergroupes (espaces localement compacts dont les mesures de Radon bornées se composent par convolution) généralisent, sous bien des aspects, les groupes localement compacts. En fait, les hypergroupes les plus naturels proviennent de l'analyse harmonique sur les groupes localement compacts par un certain type de passage au quotient: c'est ainsi qu'on étudie les algèbres de mesures centrales sur un groupe compact, ou de mesures biinvariantes par rapport à un sous-groupe compact d'un groupe localement compact, ou encore les mesures radiales sur un espace euclidien. Ces exemples, parmi d'autres, mettent en jeu la structure générale d'hypergroupe.

Divers exposés, plus ou moins complets, de la théorie des hypergroupes ont été faits récemment: voir par exemple Dunkl [1] et Spector [4], [5]. Ce n'est qu'après avoir achevé la rédaction de ce travail que nous avons eu connaissance de l'important article de Jewett [2]. Un article de Ross [3] fait le point sur les diverses présentations actuelles de la théorie, en l'absence d'une axiomatique commune aux auteurs qui traitent de ce sujet.

La notion d'hypergroupe que nous introduisons est un peu plus générale que celle de Jewett. Celui-ci exige en effet que le produit de convolution de deux mesures à support compact soit encore une mesure à support compact; nous ne formulons pas d'axiome de ce type, ce qui entraîne parfois quelques complications techniques. Bien que nous n'ayons pas d'exemple significatif d'hypergroupe qui ne satisfasse pas à la condition de compacité de Jewett, il nous a paru souhaitable de développer la théorie autant que possible sans faire appel à ce type de propriété.

$\mathrm{Au}$ centre de l'analyse harmonique classique sur un groupe localement compact se trouve la mesure de Haar. Sur un hypergroupe, la notion de mesure invariante se présente d'une façon tout aussi naturelle et joue un rôle tout aussi fondamental que dans le cas des groupes. L'unicité des mesures

Received by the editors January 22, 1976.

AMS (MOS) subject classifications (1970). Primary 22A99, 22D99, 43A10.

Key words and phrases. Hypergroup, translation operator, invariant measure, fixed point theorem.

- American Mathematical Society 1978 
invariantes, lorsqu'il en existe, et l'existence de telles mesures sur les hypergroupes compacts et les hypergroupes discrets, ont été établies par Jewett [2] et Spector [5]. Le présent travail a pour objet essentiel d'établir l'existence d'une mesure invariante sur tout hypergroupe commutatif.

Dans une première partie, nous rappelons la notion d'hypergroupe et introduisons la translation des fonctions. Puis (partie II) nous définissons les notions de mesure invariante et de mesure relativement invariante, et montrons que l'existence d'une mesure relativement invariante sur un hypergroupe entraîne celle d'une mesure invariante; nous établissons au passage des propriétés assez fortes (évidentes dans le cas des groupes) relatives aux translations dans les espaces $L^{p}$. Enfin, nous établissons dans la troisième partie de ce travail l'existence sur tout hypergroupe commutatif d'une mesure invariante à gauche; la méthode utilise d'abord le principe classique de construction d'une mesure invariante sur un groupe, telle qu'elle se trouve exposée dans le livre de Weil [6] par exemple. Mais on voit immédiatement, dans le cas des groupes, que la mesure ainsi obtenue est invariante, alors que, dans le cas général, il reste fort à faire pour obtenir une mesure qui soit effectivement invariante.

A notre connaissance, le problème de l'existence d'une mesure invariante n'est pas résolu dans le cas général d'un hypergroupe non commutatif. L'existence d'une mesure invariante sur tout hypergroupe nous semble cependant plausible et il se peut qu'un raffinement de la méthode utilisée ici dans le cas commutatif permette de traiter le cas général.

I. Notions d'hypergroupe. Translation des fonctions. Commençons par fixer les notations:

$X$ étant un espace localement compact, nous désignons par $M(X)$ l'espace de Banach des mesures de Radon complexes bornées sur $X ; M_{p}(X)$ l'ensemble des mesures de probabilité sur $X$, c'est à dire des mesures $\geqslant 0$ de masse $1 ; K(X)$ l'espace des fonctions continues à support compact sur $X$; $C_{0}(X)$ l'espace de Banach des fonctions continues sur $X$ nulles à l'infini; $C_{b}(X)$ l'espace de Banach des fonctions continues et bornées sur $X$.

Nous écrirons simplement $M, M_{p}, K, C_{0}, C_{b}$ lorsque aucune confusion ne sera à craindre. Nous noterons $M^{+}, K^{+}$, etc. les sous-ensembles des ensembles précédents formés des éléments positifs.

La mesure de masse 1 concentrée au point $x$ est notée $\delta_{x}$. Si $\mu$ est une mesure et $f$ une fonction $\mu$-integrable, l'intégrale de $f$ par rapport à $\mu$ est notée $\mu(f),\langle\mu, f\rangle$ ou $\int f d \mu$. Si $\mu$ est un mesure de $\operatorname{Radon} \operatorname{sur} X$, nous notons $S(\mu)$ son support.

Donnons maintenant la définition d'un hypergroupe:

DÉFINITION. On appelle hypergroupe un espace localement compact $X$ tel que $M(X)$ soit muni d'une structure d'algèbre de Banach par la donnée, outre la 
structure vectorielle, d'une loi de composition interne associative, notée "*" et appelée "convolution", qui vérifie les propriétés suivantes:

$\left(\mathrm{H}_{1}\right) M_{p} * M_{p} \subset M_{p}$.

$\left(\mathrm{H}_{2}\right)$ La convolution est séparément continue de $M_{p} \times M_{p}$ dans $M_{p}$ pour la topologie faible définie par la dualité entre $M$ et $C_{0}, \sigma\left(M, C_{0}\right)$.

$\left(\mathrm{H}_{3}\right)$ L'application $(x, y) \rightarrow \delta_{x} * \delta_{y}$ est continue de $X \times X$ dans $M_{p}$ muni de la topologie faible $\sigma\left(M, C_{0}\right)$.

$\left(\mathrm{H}_{4}\right)$ Il existe un point e de $X$, nécessairement unique, appelé "élément neutre de l'hypergroupe $X$ ", tel que $\delta_{e}$ soit élément neutre pour la convolution.

$\left(\mathrm{H}_{5}\right)$ Il existe un homéomorphisme involutif de $X$ sur $X$, noté $x \mapsto \check{x}$, dont le prolongement naturel à $M(X)$ vérifie $(\mu * \nu)^{\swarrow}=\check{\nu} * \check{\mu}$; en particulier $\check{e}=e$. Cet homéomorphisme sera appelé "symétrie" de l'hypergroupe $X$.

$\left(\mathrm{H}_{6}\right)$ Pour deux points $x$ et $y$ de $X$, les conditions " $x=y$ " et " $e \in$ $S\left(\delta_{x} * \delta_{y}\right)$ " sont équivalentes.

$\left(\mathrm{H}_{7}\right)$ Pour tout compact $K$ de $X$ et tout voisinage $V$ de $K$, il existe un voisinage $U$ de e tel que:

(1) $S(\mu) \subset K$ et $S(\nu) \subset U$ impliquent $S(\mu * \nu) \subset V$ et $S(\nu * \mu) \subset V$.

(2) $S(\mu) \subset K$ et $S(\nu) \subset C V$ impliquent que les supports de $\mu * \check{\nu}$, $\check{\mu} * \nu, \nu * \check{\mu}$ et $\check{\nu} * \mu$ soient disjoints de $U$.

Il est connu que, sur $M_{p}$, les topologies $\sigma\left(M, C_{0}\right)$ et $\sigma\left(M, C_{b}\right)$ coincident. De sorte que les axiomes $\left(\mathrm{H}_{2}\right)$ et $\left(\mathrm{H}_{3}\right)$ sont respectivement équivalents aux axiomes $\left(\mathrm{H}_{2}^{\prime}\right)$ et $\left(\mathrm{H}_{3}^{\prime}\right)$ ci-dessous:

$\left(\mathrm{H}_{2}^{\prime}\right)$ La convolution est séparément continue de $M_{p} \times M_{p}$ dans $M_{p}$ pour la topologie $\sigma\left(M, C_{b}\right)$.

$\left(\mathrm{H}_{3}^{\prime}\right)$ Pour toute fonction $f$ appartenant à $C_{b}$, la fonction $(x, y) \rightarrow$ $\left\langle\delta_{x} * \delta_{y}, f\right\rangle$ est continue sur $X \times X$.

Pour des exemples, voir [3].

Donnons maintenant quelques propriétés fondamentales.

THEOREME I-1. Si $\mu$ et $\nu$ appartiennent $\grave{a} M$ et $f \grave{a} C_{b}$, on a l'égalité

$$
\langle\mu * \nu, f\rangle=\iint\left\langle\delta_{x} * \delta_{y}, f\right\rangle d \mu(x) d \nu(y) .
$$

Par linéarité, il suffit de supposer $\mu$ et $\nu$ dans $M_{p}$ et $f \geqslant 0$. Alors le second membre a un sens (éventuellement $+\infty$ ) d'après $\left(\mathrm{H}_{3}^{\prime}\right)$; l'égalité s'établit d'abord lorsque $\mu$ et $\nu$ sont à supports finis, puis par un double passage à la limite en vertu de $\left(\mathrm{H}_{2}^{\prime}\right)$.

Notation. Si $A$ et $B$ sont deux parties de $X$, on désignera par $A \cdot B$ l'adhérence de la réunion des $S\left(\delta_{x} * \delta_{y}\right), x \in A, y \in B$.

THEOREME I-2. Si $\mu$ et $\nu$ appartiennent à $M(X)$, on a $S(\mu * \nu) \subset S(\mu)$. $S(\nu)$. Si $\mu$ et $\nu$ sont positives, l'inclusion est une égalité.

C'est immédiat à l'aide du Théorème I.1. 
TheOREME I-3. (1) Soient $x \in X$ et $\mu \in M$. Les propriétés suivantes sont équivalentes:

(a) $x \in S(\mu)$; (b) $e \in S\left(\delta_{\dot{x}} * \mu\right)$; (c) $e \in S\left(\mu * \delta_{\dot{x}}\right)$.

(2) Soient $x, y, z$ trois points de $X$. Les propriétés suivantes sont équivalentes:
(a) $e \in S\left(\delta_{x} * \delta_{y} * \delta_{z}\right)$;
(d) $\check{x} \in S\left(\delta_{y} * \delta_{z}\right)$;
(b) $e \in S\left(\delta_{y} * \delta_{z} * \delta_{x}\right)$;
(e) $\check{y} \in S\left(\delta_{z} * \delta_{x}\right)$;
(c) $e \in S\left(\delta_{z} * \delta_{x} * \delta_{y}\right)$;
(f) $\check{z} \in S\left(\delta_{x} * \delta_{y}\right)$.

Si $x \in S(\mu)$, alors $e \in S\left(\delta_{\dot{x}} * \mu\right)$ en vertu de $\left(\mathrm{H}_{6}\right)$ et du Théorème I.2; inversement, si $x \notin S(\mu)$, la deuxième partie de $\left(\mathrm{H}_{7}\right)$ montre qu'il existe un voisinage de $e$ disjoint du support de $\delta_{\dot{x}} * \mu$; d'où l'équivalence, dans la première partie du théorème, entre (a) et (b); de même (a) et (c) sont équivalentes. Quant à la deuxième partie du théorème, c'est un cas particulier de la première.

Remarquons que ce théorème signifie en fait que la deuxième partie de $\left(\mathrm{H}_{7}\right)$ entraîne la première.

Nous allons maintenant définir la translation des fonctions et en donner quelques propriétés.

Soit $x$ un hypergroupe.

Definition I-4. Soient $f \in C_{b}(X)$ et $\mu \in M(X)$. On appelle translatée d̀ gauche (resp. à droite) de $f$ par $\mu$ la fonction ${ }_{\mu}$ (resp. $f_{\mu}$ ) définie sur $X$ par

$$
{ }_{\mu} f(x)=\left\langle\check{\mu} * \delta_{x}, f\right\rangle \quad\left(\text { resp. } f_{\mu}(x)=\left\langle\delta_{x} * \check{\mu}, f\right\rangle\right) .
$$

$\mathrm{Si}, a$ étant un point de $X, \mu$ est la mesure $\delta_{a}$, on écrira simplement ${ }_{a} f$ et $f_{a}$ au lieu de $\delta_{a} f$ et $f_{\delta_{a}}$.

THEOREME I.5. Les fonctions ${ }_{\mu} f$ et $f_{\mu}$ appartiennent $\dot{a} C_{b}$ et leurs normes sont majorées par $\|\mu\|\|f\|$.

La continuité des translatées est une conséquence immédiate de $\left(\mathrm{H}_{2}^{\prime}\right)$, et la relation entre les normes est évidente $\left({ }^{1}\right)$.

Remarque. On en déduit que la convolution est séparément continue non seulement de $M_{p} \times M_{p}$ dans $M_{p}$, mais de $M \times M$ dans $M$ pour la topologie $\sigma\left(M, C_{b}\right)$.

Theoreme I.6. Soient $f \in C_{b}$, $\mu$ et $\nu \in M$. Alors

$$
\left\langle\nu,{ }_{\mu} f\right\rangle=\langle\check{\mu} * \nu, f\rangle \text { et }\left\langle\nu, f_{\mu}\right\rangle=\langle\nu * \check{\mu} f\rangle \text {. }
$$

Cela résulte immédiatement du Théorème I.i.

(')Dans (2) et (3), nous avions affirmé par erreur que ${ }_{\mu} f$ et $f_{\mu}$ appartenaient à $C_{0}$ si $f$ appartenait à $C_{0}$. On en déduisait la propriété probablement fausse - encore que vraie dans les cas particuliers connus-selon laquelle la convolution était séparément continue de $M \times M$ dans $M$ pour la topologie faible $\sigma\left(M, C_{0}\right)$. 
THEOREME I.7. Soient $f \in C_{b}, \mu$ et $\nu \in M$. Alors

$$
{ }_{\mu * \nu} f={ }_{\mu}\left({ }_{w} f\right) \text { et } f_{\mu * \nu}=\left(f_{\mu}\right)_{\nu} .
$$

Conséquence immédiate du théorème précédent.

Theoreme I.8. Soient $f \in C_{b}$ et $\mu \in M$. Alors (en désignant par $S(\phi)$ le support d'une fonction continue $\phi)$

$$
S\left({ }_{\mu} f\right) \subset S(\mu) \cdot S(f) \text { et } \quad S\left(f_{\mu}\right) \subset S(f) \cdot S(\mu) ;
$$

les inclusions sont des égalités si f et $\mu$ sont positives.

Montrons par exemple la propriété relative à ${ }_{\mu} f$.

Si $x \notin S(\mu) \cdot S(f)$, pour tout point $y$ de $S(\mu)$ et tout point $z$ de $S(f), x$ n'appartient pas à $S\left(\delta_{y} * \delta_{z}\right)$ et, d'après I.3, $\check{z} \notin S\left(\delta_{\dot{x}} * \delta_{y}\right)$, ou encore $z \notin$ $S\left(\delta_{y} * \delta_{x}\right)$; dans ce cas $\left\langle\delta_{y} * \delta_{x}, f\right\rangle=0$ pour tout $y$ du support de $\mu$ et, puisque $\left\langle\check{\mu} * \delta_{x}, f\right\rangle=\int\left\langle\delta_{\check{y}} * \delta_{x}, f\right\rangle d \mu(y)$ d'après I.1, cette quantité, égale à ${ }_{\mu} f(x)$, est nulle. D'où l'inclusion cherchée.

Pour la suite, nous aurons besoin d'un lemme.

Lemme I.9. Soient $A$ et $B$ deux parties de X. Alors

$$
A \cdot B=\bar{A} \cdot B=A \cdot \bar{B}=\bar{A} \cdot \bar{B} .
$$

(Rappelons que, par définition, $A \cdot B$ est fermé.) Il suffit d'établir l'une des égalités, par exemple $A \cdot B=\bar{A} \cdot \bar{B}$; l'inclusion $A \cdot B \subset \bar{A} \cdot \bar{B}$ est évidente; inversement, soit $x$ un point de $X$ tel que $x \notin A \cdot B$; il exis te donc une fonction $u \in C_{b}^{+}$telle que $u(x)>0$ et qui est nulle sur $A \cdot B$; donc, pour tout $t \in A$ et tout $z \in B,\left\langle\delta_{t} * \delta_{z}, u\right\rangle=0$; comme cette fonction des deux variables $t$ et $z$ est continue par $\left(\mathrm{H}_{3}^{\prime}\right)$, il en résulte que $\left\langle\delta_{t} * \delta_{z}, u\right\rangle=0$ si $t \in \bar{A}$ et $z \in \bar{B}$; donc $u$ est nulle sur $\bar{A} \cdot \bar{B}$, et $x$ ne peut appartenir à cet ensemble.

Revenons alors au support de ${ }_{\mu} f$ dans le cas où $f$ et $\mu$ sont positives, en éliminant le cas trivial $f \equiv 0$. Soit $A$ l'ensemble des points où $f$ est $>0$ : $S(f)=\bar{A}$ et, d'après le lemme ci-dessus, $S(\mu) \cdot S(f)=S(\mu) \cdot A$; soit donc $x$ un point appartenant à $S(\mu) \cdot A$ : pour tout voisinage $U$ de $x$, il existe $y \in S(\mu)$ et $z \in A$ tels que $S\left(\delta_{y} * \delta_{z}\right) \cap U \neq \varnothing$; soit $t$ un point de cette intersection; d'après I.3, $z \in S\left(\delta_{\dot{y}} * \delta_{t}\right)$, donc $\left\langle\delta_{\dot{y}} * \delta_{t}, f\right\rangle$, qui n'est autre que $f_{i}(\check{y})$ est $>0$; comme la fonction positive $y \mapsto f_{i}(\check{y})$ est' continue et que $y \in S(\mu)$, il en résulte que

$$
\begin{aligned}
\int f_{i}(\check{y}) d \mu(y) & =\int\left\langle\delta_{\check{y}} * \delta_{t}, f\right\rangle d \mu(y)=\left\langle\check{\mu} * \delta_{t}, f\right\rangle \\
& ={ }_{\mu} f(t)>0,
\end{aligned}
$$

donc ${ }_{\mu} f$ n'est pas identiquement nulle sur $U$, ce qui prouve que $x$ appartient au support de ${ }_{\mu} f$, puisque $U$ était un voisinage quelconque de $x$. 
II. Mesures invariantes et mesures relativement invariantes. Soit $X$ un hypergroupe.

DEFINITION II.1. On appelle mesure invariante à gauche sur $X$ une mesure de Radon (non nécessairement bornée) positive non nulle $m$ telle que, pour toute $f \in K(X)$ et tout $a \in X$, la fonction af soit $m$-intégrable et que $m\left({ }_{a} f\right)=m(f)$

DEFINITION II.2. On appelle mesure relativement invariante à gauche sur $X$ une mesure de Radon positive non nulle $m$ telle qu'il existe une fonction $k$ strictement positive définie sur $X$ de telle sorte que la propriété suivante soit vérifiée: pour toute $f \in K(X)$ et tout $a \in X$, la fonction af est m-intégrable et $m\left({ }_{a} f\right)=k(a) m(f)$. La fonction $k$ est appelée multiplicateur de la mesure relativement invariante $m$.

On définirait de manière analogue les mesures (relativement) invariantes à droite.

Pour une fonction $f$, soit $\check{f}$ la fonction définie sur $X$ par $\check{f}(x)=f(\check{x})$. Compte tenu de la relation immédiate ${ }_{\mu} \check{f}=\left(f_{\dot{\mu}}\right)^{\circ}$, où $f \in K(X)$ et $\mu \in$ $M(X)$, on voit que si $m$ est une mesure relativement invariante à gauche de multiplicateur $k, \check{m}$ est relativement invariante à droite de multiplicateur $\check{k}$.

THEOREME II.3. Soit $m$ une mesure relativement invariante $\dot{a}$ gauche de multiplicateur $k$.

(1) Le support de $m$ est $X$.

(2) $k$ est une fonction continue.

(3) Pour une mesure de Radon bornée positive $\mu$, les trois propriétés ci-dessous sont équivalentes:

(a) il existe $f \in K^{+}$, telle que ${ }_{\mu} f$ soit $m$-intégrable, $f \neq 0$;

(b) pour toute $f \in C_{b}$ m-intégrable, ${ }_{\mu} f$ est $m$-intégrable;

(c) $k$ est $\mu$-intégrable.

Dans ce cas, pour $f \in C_{b}$ m-intégrable, $m\left({ }_{\mu} f\right)=\mu(k) m(f)$.

(4) $S i \mu$ et $\nu$ sont deux mesures bornées par rapport auxquelles $k$ est intégrable, $k$ est $\mu * \nu$ intégrable et $(\mu * \nu)(k)=\mu(k) \nu(k)$.

Remarquons d'abord qu'une mesure invariante n'est autre qu'une mesure relativement invariante de multiplicateur constant égal à 1 . De sorte que, en particulier, la 3ième partie du théorème montre que, pour $f \in K(X)$ et $\mu \in M(X),{ }_{\mu} f$ est intégrable par rapport à une mesure $m$ invariante à gauche et que $m\left({ }_{\mu} f\right)=\left(\int d \mu\right) m(f)$.

Soit $a$ un point du support de la mesure relativement invariante à gauche $m$ et soit $b$ un point quelconque de $X$. Montrons que $b$ appartient au support de $m$. Soit $f$ une fonction de $K^{+}(X)$, telle que $f(b)>0$. Montrons que $m(f)$ est $>0$ : la fonction $\not{b} f$ prend en $e$ la valeur $f(b)>0$, et $m(\breve{b} f)=k(\breve{b}) m(f)$ de sorte qu'il suffit de montrer que $e$ appartient au support de $m$; soit $g$ une fonction de $K^{+}(X)$, telle que $g(e)$ soit $>0$ : alors ${ }_{a} g$ prend en $a$ la valeur 
$\left\langle\delta_{a} * \delta_{a}, g\right\rangle$ qui est $>0$ puisque $e \in S\left(\delta_{\dot{a}} * \delta_{a}\right)$ : donc $m\left({ }_{a} g\right)=k(a) m(g)>$ 0 ; ceci prouve donc que $m(f)$ est $>0$, donc que $b$ appartient au support de $m$ : la première partie du théorème est établie.

Soient maintenant $a$ un point de $X, A$ un voisinage compact de $a, B$ un voisinage compact de $A$. D'après $\left(\mathrm{H}_{7}\right)$, il existe un voisinage $U$ de $e$ tel que $A \cdot U \subset B$, de sorte que, si $f \in K^{+}(X)$ est à support dans $U, S\left({ }_{a} f\right) \subset B$ pour tout $a \in A$ d'après le théorème I.8. Dans ces conditions, l'application $a \mapsto{ }_{a} f$ est continue de $A$ dans $K(B)$ : s'il n'en était pas ainsi il existerait un nombre $\alpha>0$, un point $b$ de $A$, et pour tout voisinage $V$ de $b$ dans $A$, un point $b_{V}$ de $A$ et un point $c_{V}$ de $B$ tels que $\left|{ }_{b_{V}} f\left(c_{V}\right)-{ }_{b} f\left(c_{V}\right)\right|>\alpha$. $B$ étant compact, il existe un point $c$ de $B$ tel que, pour tout voisinage $V$ de $b$ et tout voisinage $W$ de $c$, il existe un voisinage $V^{\prime}$ de $b$ contenu dans $V$ tel que $c_{V^{\prime}} \in W$; donc sur $V \times W$, la fonction $(x, y) \mapsto\left\langle\delta_{\dot{x}} * \delta_{y}, f\right\rangle$ a une oscillation supérieure à $\alpha$; comme ces ensembles $V \times W$ constituent un système fondamental de voisinages de $(b, c)$ dans $A \times B$, cette fonction n'est pas continue en $(b, c)$, ce qui contredit l'axiome $\left(\mathrm{H}_{3}\right)$. Alors, puisque pour $a \in A$ les fonctions $f$ ont leurs supports dans un compact fixe, l'application $a \mapsto m\left({ }_{a} f\right)$ est continue, donc la fonction $k$ est continue sur $A$ (prendre $f \not \equiv 0$ ), donc $k$ est continue sur $X$ car $A$ est un voisinage du point quelconque $a$. D'où la deuxième partie.

Soient maintenant $f \in C_{b}^{+}$et $\mu \in M^{+}$. On a alors les égalités suivantes (où les termes écrits prennent éventuellement la valeur $+\infty$ ), $g(x, y)$ désignant la fonction, continue sur $X \times X,\left\langle\delta_{\check{x}} * \delta_{y}, f\right\rangle={ }_{x} f(y)$ :

$$
\begin{aligned}
\int_{\mu} f(y) d m(y) & =\int d m(y) \int g(x, y) d \mu(x)=\int d \mu(x) \int g(x, y) d m(y) \\
& =\int d \mu(x) \int_{x} f(y) d m(y)=\int k(x) d \mu(x) \int f(y) d m(y) .
\end{aligned}
$$

De là découle la partie 3 du théorème.

Quant à la 4ième partie, elle est immediate: soit $f \in K^{+}(X)$ non identiquement nulle, donc telle que $m(f)>0$. Si $\mu$ et $\nu$ sont deux mesures par rapport auxquelles $k$ est intégrable, on peut écrire, en vertu de la 3ième partie, que ${ }_{w} f$ est $m$-intégrable, donc aussi ${ }_{\mu}\left({ }_{n}\right)={ }_{\mu \cdot} f$, et que

$$
m\left({ }_{\mu * v} f\right)=(\mu * \nu)(k) m(f)=m\left({ }_{\mu}\left({ }_{n} f\right)\right)=\mu(k) m\left({ }_{k} f\right)=\mu(k) \nu(k) m(f),
$$

d'où le résultat.

Venons-en maintenant au théorème fondamental de cette partie.

TheOREME Fondamental II.4. Soient $X$ un hypergroupe, $m$ une mesure relativement invariante à gauche sur $X$ de multiplicateur $k$. Alors

(1) la mesure $m / k$ est invariante à gauche;

(2) pour toute $f \in K(X)$, toute $\phi \in C_{b}(X)$ et toute mesure $\mu \in M(X)$, 


$$
\int\left\{{ }_{\mu} f(x)\right\} \phi(x) \frac{d m(x)}{k(x)}=\int f(x)\left\{{ }_{\mu} \phi(x)\right\} \frac{d m(x)}{k(x)} .
$$

Remarquons que ce théorème est immédiat si $X$ est un groupe. La difficulté dans le cas général d'un hypergroupe provient de ce que, pour $a \in X$ et $f, \phi$ dans $K$ ou $C_{b}, \quad \dot{a}\left({ }_{a} f\right) \neq f$ et ${ }_{a}(f \phi) \neq\left({ }_{a} f\right)\left({ }_{a} \phi\right)$ en général.

La démonstration se réduit à celle de la deuxième partie du théorème; la première partie en découle en effet (il suffit de prendre $\phi \equiv 1$ ).

Nous aurons besoin de quatre lemmes.

LEMME II.5. Soient $\nu$ une mesure de Radon bornée par rapport à laquelle $k$ est intégrable, et $g \in C_{b}(X)$. Pour tout $\varepsilon>0$, il existe un voisinage $U$ de $\dot{e}$ tel que, pour toute fonction $h$ de $K^{+} \grave{a}$ support dans $U$, d'intégrale 1 par rapport à $m$, on ait

$$
\left|\int\left({ }_{\nu} h\right) g d m-\nu(k g)\right|<\varepsilon .
$$

Il suffit évidemment d'établir ce résultat pour $\nu$ et $g \geqslant 0$.

Soit d'abord une décomposition de $\nu$ sous la forme $\nu_{1}+\nu_{2}: \nu_{1}$ et $\nu_{2}$ étant positives, on a évidemment

$$
\left|\int\left({ }_{\nu} h\right) g d m-\nu(k g)\right| \leqslant\left|\int\left({ }_{\nu_{1}} h\right) g d m-\nu_{1}(k g)\right|+\left|\int\left({ }_{\nu_{2}} h\right) g d m-\nu_{2}(k g)\right|
$$

et, puisque $\int h d m=1$, le deuxième terme du membre de droite de l'inégalité ci-dessus est majoré par $2 v_{2}(k)\|g\|$. Comme on peut choisir $\nu_{1}$ à support compact et $\nu_{2}$ telle que $\nu_{2}(k)$ soit arbitrairement petite, on voit qu'il suffit d'établir le résultat pour une mesure $\nu$ à support compact.

Soient alors $A$ le support compact de $\nu, B$ un voisinage compact de $A$. Pour tout nombre positif $\eta$, il existe des ouverts relativement compacts $V_{j}$ et $W_{j}$ $(1 \leqslant j \leqslant n)$ tels que $\bar{W}_{j} \subset V_{j}, B \subset \cup_{j} W_{j}$ et que sur chacun des $V_{j}$ l'oscillation de $g$ soit inférieure à $\eta$. D'après $\left(\mathrm{H}_{7}\right)$, il existe un voisinage $U$ de $e$ tel que, pour tout $j, W_{j} \cdot U \subset V_{j}$. On peut écrire $\nu=\sum_{j=1}^{n} \nu_{j}$, les $\nu_{j}$ étant positives et à support dans $W_{j}$, de sorte que, pour une fonction $h$ continue à support compact dans $U,{ }_{v_{j}} h$ est nulle hors de $V_{j}$, d'après le Théorème I.8. Par ailleurs,

$$
\left|\int\left({ }_{\nu} h\right) g d m-\nu(k g)\right| \leqslant \sum_{j}\left|\int\left({ }_{\nu_{j}} h\right) g d m-\nu_{j}(k g)\right| .
$$

Soit, pour tout $j, x_{j}$ un point de $V_{j}$. Les termes du second membre sont alors, en vertu des choix des $W_{j}, V_{j}$ et $U$, majorés par

$$
\left|g\left(x_{j}\right) \int_{\nu_{j}} h d m-g\left(x_{j}\right) \nu_{j}(k)\right|+\eta \int_{\nu_{j}} h d m+\eta v_{j}(k)=2 \eta v_{j}(k)
$$


(d'après le Théorème II.3), de sorte que $\left|\int\left({ }_{\nu} h\right) g d m-\nu(k g)\right|<2 \eta \nu(k)$ ce qui établit le lemme, en posant $\eta=\varepsilon / 2 \nu(k)$.

Soit maintenant $\mu$ une mesure par rapport à laquelle $k$ est intégrable ( $\mu$ à support compact par exemple). L'application $f \mapsto{ }_{\mu} f$ dèfinie de $K$ dans $L^{1}(X, m)$ se prolonge (en vertu du Théorème II.3) en une application continue de $L^{1}(X, m)$ dans $L^{1}(X, m)$, que nous noterons $\lambda_{\mu}$. Soit $L_{\mu}$ l'application transposée de $\lambda_{\mu}$, de $L^{\infty}(X, m)$ dans $L^{\infty}(X, m)$. Si $f \in K$ et si $\phi$ est une fonction continue bornée ainsi que $\phi / k(\phi \in K$ par exemple), on a

$$
\int\left({ }_{\mu} f\right) \frac{\phi}{k} d m=\int f \cdot L_{\mu}\left(\frac{\phi}{k}\right)=\int f \cdot k L_{\mu}\left(\frac{\phi}{k}\right) \frac{d m}{k},
$$

de sorte qu'il s'agit d'abord de montrer que, pour une telle fonction $\phi$, ${ }_{\mu} \phi=k L_{\mu}(\phi / k)$.

Nous allons montrer que, pour une fonction $g \in C_{b}$, on a $L_{\mu}(g)=$ $k^{-1}{ }_{\mu}(k g)$, le second membre de cette égalité ayant un sens de manière naturelle, d'où le résultat cherché en posant $g=\phi / k$, pour $\phi \in K$.

LEMME II.6. Pour toute $g \in C_{b}(K)$ et toute $\nu \in M(X)$ par rapport à laquelle $k$ est intégrable, $L_{\nu}(g)$ est une fonction $m$-presque partout égale à une fonction continue en e, prenant en e la valeur $\nu(\mathrm{kg})$.

Cette propriété est en effet équivalente à l'existence, pour tout $\varepsilon>0$, d'un voisinage $U$ de $e$ tel que, pour toute $h \in K^{+}(X)$ à support dans $U$, d'intégrale 1 par rapport à $m$, on ait

$$
\left|\int h L_{\nu}(g) d m-\nu(k g)\right|<\varepsilon .
$$

Or cette relation s'écrit encore $\left|\int\left({ }_{\nu} h\right) g d m-\nu(k g)\right|<\varepsilon$ et résulte du Lemme II.5, dont le Lemme II.6 n'est donc qu'une reformulation.

LEMME II.7. Soient $u \in K(X)$, $v$ la mesure um et $B$ un voisinage compact $d u$ support de $u$. Pour tout $\varepsilon$ positif, il existe un voisinage $U$ de e tel que, pour toute fonction $h \in K^{+} \grave{a}$ support dans $U$, d'intégrale 1 par rapport $\grave{a} m$, on ait $\left\|{ }_{\nu} \check{h}-k u\right\|<\varepsilon$ et que la fonction, $\check{\text {, }}$ soit à support dans $B$.

Soient $A$ le support compact de $u, B$ un voisinage compact de $A$. Pour un nombre positif $\eta$, il existe des ouverts relativement compacts $V_{j}$ et $W_{j}$ $(1<j \leqslant n)$ tels que $\bar{W}_{j} \subset V_{j}$, que $B \subset \cup W_{j}$ et que sur chacun des $V_{j}$ l'oscillation de $u$ soit inférieure à $\eta$. D'après $\left(\mathrm{H}_{7}\right)$, il existe un voisinage $U$ de $e$ tel que, pour tout $j,\left(\bar{W}_{j} \cdot C V_{j}\right) \cap U=\varnothing$ et que $A \cdot U \subset B$.

Alors, pour $h \in K^{+}$à support dans $U$, la fonction $\check{h}$ est à support dans $U$ (à condition de prendre, ce qui est évidemment possible, $U$ symétrique, c'est-à-dire $U$ égale à $\check{U}$ ) et la fonction ${ }_{\nu} \check{h}$ s'annule hors de $B$. Il suffit donc d'assurer l'inégalité $\left|{ }_{\nu} \check{h}(x)-k(x) u(x)\right|<\varepsilon$ pour tout $x$ de $B$. Or, si $x \in B$, 
soit $j$ tel que $x \in W_{j}$; alors

$$
\begin{aligned}
\check{\nu}(x) & =\int\left\langle\delta_{\dot{y}} * \delta_{x}, \check{h}\right\rangle u(y) d m(y)=\int\left\langle\delta_{\dot{x}} * \delta_{y}, h\right\rangle u(y) d m(y) \\
& =\int_{V_{j}}\left\langle\delta_{\dot{x}} * \delta_{y}, h\right\rangle u(y) d m(y)=\int x_{x} h(y) u(y) d m(y) \\
& =\int_{V_{j}} x h(y) u(y) d m(y),
\end{aligned}
$$

et on a

$$
\left|{ }_{\nu} \check{h}(x)-u(x) k(x)\right|<\eta k(x),
$$

de sorte qu'il suffit de prendre $\eta=\varepsilon / \sup _{B} k$.

LEMme II.8. Soit $p$ une fonction de $L^{\infty}(X, m)$ telle que, pour toute mesure $\nu$ de la forme um avec $u \in K, L_{v}(p)$ soit m-presque partout égale à une fonction continue en e et nulle en e. Alors $p=0 \mathrm{~m}$-localement presque partout.

En effet, soient $\varepsilon>0$ et $\nu=u m$ fixée. L'hypothèse exprime l'existence d'un voisinage $U$ de $e$ tel que, pour toute $h \in K^{+}$à support dans $U$, d'intégrale 1 par rapport à $m$, on ait $\left|\int L_{\nu}(p) h d m\right|<\varepsilon$.

Or $\int L_{\nu}(p) h d m=\int\left({ }_{v} h\right) p d m$, et, à condition de prendre $U$ assez petit et $h$ paire $(h=\check{h})$, ceci entraîne, en vertu du Lemme II.7, que $\left|\int k u p d m\right|<\varepsilon+$ $\varepsilon\|p\| m(B)$, où $B$ désigne un voisinage compact du support de $u$ indépendant de $\varepsilon$. Donc, pour toute $u \in K(X)$ on a $\int k u p d m=0$, ce qui entraîne bien l'égalité $p=0 m$-localement presque partout.

Nous sommes maintenant en mesure d'établir le théorème. Il suffit évidemment de raisonner en supposant que les fonctions $f$ et $\phi$, ainsi que la mesure $\mu$, sont positives.

Soit donc $f \in K^{+}$. Prenons d'abord $\phi \in K^{+}$et $\mu \in M^{+}$telle que $k$ soit $\mu$-intégrable. Pour toute mesure $\nu=u m$, avec $u \in K(X)$, on a, en posant $g=\phi / k, L_{\nu} L_{\mu}(g)=L_{\mu, \nu}(g)$. D'après II.6, c'est une fonction presque partout égale à une fonction continue en $e$, prenant en ce point la valeur $\langle\mu * \nu, k g\rangle$. D'autre part, la fonction $k^{-1}{ }_{\dot{\mu}}(k g)$ est continue bornée: en effet, $\check{\mu}(k g)(x)=\left\langle\mu * \delta_{x}, k g\right\rangle$ est positif ou nul et majoré par $\|g\|\left\langle\mu * \delta_{x}, k\right\rangle$, soit d'après le Théorème II.3, $\|g\| \mu(k) k(x)$; le Lemme II.6 appliqué à la fonction $k^{-1} \cdot \dot{\mu}(k g)$ montre donc que $L_{\nu}\left(k^{-1} \dot{\mu}(k g)\right)$ est presque partout égaie à une fonction continue en $e$, prenant en ce point la valeur $\langle\nu, \dot{\mu}(k g)\rangle=$ $\langle\mu * \nu, k g\rangle$. Le résultat cherché découle alors du Lemme II.8 appliqué à la fonction $L_{\mu}(g)-k^{-1}{ }_{\mu}(k g)$, élément de $L^{\infty}(X, m)$. En fait, il suffit ici que $\phi \in C_{b}$, ainsi que $g=\phi / k$.

Désignons maintenant par $m_{0}$ la mesure $m / k$. Nous avons donc montré que, en particulier si $f$ et $\phi$ appartiennent à $K^{+}(X)$, et $a \in X$, 


$$
\int\left({ }_{a} f\right) \phi d m_{0}=\int f\left({ }_{a} \phi\right) d m_{0} .
$$

Lemme II.9. Si $A$ et $B$ sont deux compacts de $X, A \cdot B$ est dénombrable à l'infini.

Il suffit de l'établir pour deux compacts contenant $A$ et $B$, par exemple pour deux compacts $A^{\prime}$ et $B^{\prime}$ qui soient les adhérences d'ouverts contenant $A$ et $B$. Or deux tels compacts sont les supports de mesures positives $\alpha$ et $\beta$ (cela découle du Théorème II.3 lorsque $X$ porte une mesure relativement invariante; c'est vrai plus généralement s'il existe sur $X$ une mesure positive dont le support soit $X$, ce qui est vérifié dans tous les cas, comme on le verra à la partie III), donc $A \cdot B$ est contenu dans $A^{\prime} \cdot B^{\prime}$ qui est $\sigma$-compact, puisque c'est le support de la mesure bornée $\alpha * \beta$.

Soient maintenant $a \in X, f \in K^{+}, \phi \in C_{b}^{+}$. Si $A$ est le support de $f$, d'après le lemme ci-dessus, $(\{a\} \cdot A) \cup A$ est contenu dans la réunion d'une suite $K_{n}$ de compacts, tels que $K_{n} \subset K_{n+1}^{0}$. Soient $u_{n}$ des fonctions de $K^{+}$, à valeurs entre 0 et 1 , telles que $u_{n}=1$ sur $K_{n}$, et 0 hors de $K_{n+1}$. Alors la suite $u_{n} \phi$ tend en croissant vers $\phi$, et d'après le théorème de convergence monotone de Lebesgue, $a\left(u_{n} \phi\right)$ tend en croissant vers ${ }_{a} \phi$ sur l'ensemble $(\{a\} \cdot A) \cup A$ qui contient les supports de $f$ et de $f$ (Théorème I.8). On a donc, en appliquant une fois encore ce théorème de convergence monotone,

$$
\begin{aligned}
\int\left({ }_{a} f\right) \phi d m_{0} & =\lim _{n} \int\left({ }_{a} f\right)\left(u_{n} \phi\right) d m_{0}=\lim _{n} \int f_{\dot{a}}\left(u_{n} \phi\right) d m_{0} \\
& =\int f_{\dot{a}} \phi d m_{0} .
\end{aligned}
$$

En prenant $\phi \equiv 1$, on voit que $m_{0}$ est une mesure invariante à gauche. La relation générale $\int\left({ }_{\mu} f\right) \phi d m_{0}=\int f\left({ }_{\mu} \phi\right) d m_{0}$ valable pour $f \in K, \phi \in C_{b}$, $\mu \in M$, résulte aussitôt du cas particulier, établi plus haut, où $\mu$ est une mesure ponctuelle.

THEOREME II.10. Soient $m$ une mesure relativement invariante à gauche, $k$ son multiplicateur, $\mu$ une mesure $\in M_{p}$ par rapport à laquelle $k$ soit intégrable.

(1) Les opérateurs $\lambda_{\mu}: f \mapsto{ }_{\mu} f$ et $L_{\mu}: f \mapsto k^{-1}{ }_{\mu}(k f)$ définis sur $K$ se prolongent de manière unique en des opérateurs linéaires continus de $L^{1}(X, m)$ dans $L^{1}(X, m)$, que nous noterons encore $\lambda_{\mu}$ et $L_{\mu}$, de normes $\mu(k)$ et 1 respectivement.

(2) Les opérateurs $\lambda_{\mu}$ et $L_{\mu}$ définis initialement sur $C_{b}$ admettent des prolongements en des opérateurs linéaires continus de $L^{\infty}(X, m)$ dans $L^{\infty}(X, m)$, que nous noterons encore $\lambda_{\mu}$ et $L_{\mu}$, tels que $\lambda_{\mu}$ (resp. $L_{\mu}$ ) sur $L^{\infty}$ soit le transposé de $L_{\mu}$ (resp. $\lambda_{\mu}$ ) sur $L^{1}$. Les normes de $\lambda_{\mu}$ et $L_{\mu}$ sont dans ce cas 1 et $\mu(k)$ respectivement.

(3) De même, $\lambda_{\mu}$ et $L_{\mu}$ définis sur $K$ admettent, pour tout réel $p \in[1,+\infty[$, 
des prolongements linéaires continus uniques, encore notés $\lambda_{\mu}$ et $L_{\mu}$, de $L^{p}(X, m)$ dans $L^{p}(X, m)$, avec coincidence sur les intersections des divers espaces $L^{p}$. Leurs normes sont majorées par $\mu(k)^{1 / p}$ et $(\mu(k))^{1-1 / p}$ respectivement.

(4) Soient $p, q \in[1,+\infty]$, avec $1 / p+1 / q=1, f \in L^{p}(X, m)$ et $g \in$ $L^{q}(X, m)$. Alors, en posant ${ }_{\mu} f=\lambda_{\mu}(f)$ et ${ }_{\dot{\mu}}(k g)=k L_{\mu}(g)$, on a

$$
\int\left({ }_{\mu} f\right) g d m=\int f \cdot k^{-1} \cdot{ }_{\mu}(k g) d m \text {. }
$$

Pour le (1), la propriété concernant $\lambda_{\mu}$ est évidente (on l'a déjà utilisée); pour $L_{\mu}$, il suffit de vérifier que, pour toute $f \in K^{+},\left\|k^{-1}{ }_{\dot{\mu}}(k f)\right\|_{1}=\|f\|_{1}$. Or cela résulte du fait que la mesure $m / k$ est invariante appliqué à la fonction $k f$. L'assertion concernant les normes d'opérateurs est immédiate.

Dans la deuxième partie du théorème, la propriété concernant le prolongement de $L_{\mu}$ à $L^{\infty}$ résulte du Théorème II.4; pour le prolongement de $\lambda_{\mu} \grave{a} L^{\infty}$, il suffit de vérifier que pour toute $f \in C_{b}$ et toute $\phi \in K$ on a

$$
\int\left({ }_{\mu} f\right) \phi d m=\int f k^{-1}{ }_{\mu}(k \phi) d m,
$$

ci qui résulte encore de l'invariance de la mesure $m / k$ et du Théorème II.4.

La 3ième partie résulte de ce qui précède par le théorème d'interpolation de Riesz-Thorin. La cohérence des prolongements est banale.

Quant à la 4ième partie, elle est déjà connue lorsque $f$ et $\phi$ sont à supports compacts, donc pour $f \in L^{p}$ et $g \in L^{q}$ avec $\left.p, q \in\right] 1, \infty[$ par continuité, et le résultat est également vrai pour $p=1, q=+\infty$ (ou l'inverse) par définition des opérateurs $\lambda_{\mu}$ et $L_{\mu}$ sur $L^{\infty}$.

THEOREME II.11. Si $m$ est une mesure invariante, et $f \in L^{1}(X, m)$, on a $\left({ }_{\mu} f\right) m=\mu *(f m)$.

En effet, pour $g \in K$ quelconque.

$$
\begin{aligned}
\left\langle\left({ }_{\mu} f\right) m, g\right\rangle & =\int g(x)_{\mu} f(x) d m(x)=\iint\left\langle\delta_{y} * \delta_{x}, f\right\rangle g(x) d m(x) d \mu(y) \\
& =\int d \mu(y) \int_{y} f(x) g(x) d m(x)=\int d \mu(y) \int f(x)_{y} g(x) d m(x) \\
& =\iint\left\langle\delta_{y} * \delta_{x}, g\right\rangle f(x) d m(x) d \mu(y)=\langle\mu * f m, g\rangle
\end{aligned}
$$

d'après les Théorèmes II.4 et I.1.

THEOREME II.12. Soient $m$ une mesure invariante à gauche. Si $f$ et $g \in$ $L^{1}(X, m)$, la mesure bornée $(\mathrm{fm}) *(\mathrm{gm})$ est absolument continue par rapport à $m$ et admet pour densité une fonction m-intégrable notée $f * g$, appelée produit 
de convolution de $f$ et de $g$, et définie pour presque tout $x$ par $(f * g)(x)=$ $\int f(y){ }_{y} g(x) d m(y)$.

On a en effet, pour $h \in K(X)$ quelconque,

$$
\begin{aligned}
\langle f m * g m, h\rangle & =\iint\left\langle\delta_{y} * \delta_{x}, h\right\rangle f(y) g(x) d m(y) d m(x) \\
& =\int f(y) d m(y) \int_{y} h(x) g(x) d m(x) \\
& =\int f(y) d m(y) \int h(x)_{y} g(x) d m(x) \\
& =\int h(x) d m(x) \int f(y)_{y} g(x) d m(y) .
\end{aligned}
$$

Remarque. Il résulte $d u$ théorème ci-dessus que la convolution munit $L^{1}(X, m)$ d'une structure d'algèbre de Banach. En fait l'injection isométrique $f \mapsto f m$ de $L^{1}(X, m)$ dans $M(X)$ identifie $L^{1}$ à un idéal à gauche de $M$, puisque $\dot{\mu} *(\mathrm{fm})=\left({ }_{\mu} f\right) m$ est alors identifiée $\grave{a}{ }_{\mu} f\left(f \in L^{1}, \mu \in M\right)$. On pourra dire désormais que la fonction translatée ${ }_{\mu} f$ est le produit de convolution de la mesure $\mu$ et de la fonction $f$.

III. Construction d'une mesure invariante sur tout hypergroupe commutatif. Nous allons montrer que tout hypergroupe commutatif possède une mesure relativement invariante d'où, en vertu du Théorème II.4, l'existence d'une mesure invariante.

Une première étape consiste à construire en nous inspirant de la méthode classique de Weil [6], une mesure "sous-invariante à gauche" sur l'hypergroupe $X$ - non nécessairement commutatif pour le moment c'est-à-dire d'une mesure $\sigma$ telle que, pour toute $f \in K^{+}(X)$ et toute $\mu \in M_{p}(X),{ }_{\mu} f$ soit $\sigma$-intégrable et que $0<\sigma\left(\left({ }_{\mu} f\right)\right) \leqslant \sigma(f)$ si $f \neq 0$.

A. Soit $X$ un hypergroupe quelconque. Désignons par $L^{+}$l'ensemble des fonctions qui s'écrivent comme sommes finites de fonctions du type ${ }_{\alpha} f$, avec $f \in K^{+}$et $\alpha \in M^{+}$; soit $L$ l'espace vectoriel de fonctions engendré par $L^{+}$: c'est un sous-espace de $C_{b}$ invariant par les translations à gauche.

Si $f \in C_{b}^{+}$et $g \in K^{+}$, il existe (lorsque $f$ est $\neq 0$ ) une mesure $\mu \in M^{+}$ telle que $g \leqslant{ }_{\mu} f$ (en effet, pour tout point $x$, une translatée de $f$ par une mesure positive bien choisie est $>0$ en $x$; la compacité du support de $g$ permet de conclure en prenant une combinaison linéaire à coefficients positifs de telles mesures), et il en est de même si $g$ est de la forme ${ }_{\alpha} h$ avec $h \in K^{+}$ et $\alpha \in M^{+}$, et aussi si $g \in L^{+}$. Notons que, dans ces conditions, $\|g\|<$ $\|\mu\|\|f\|$.

Pour deux fonctions $f$ et $g \in L^{+}$, posons, si $f \neq 0$,

$$
A_{f}(g)=\inf \|\mu\|, \quad \mu \in M^{+}, \quad g \leqslant{ }_{\mu} f .
$$


Les relations suivantes sont immédiates pour $f, g, h \in L^{+}$et $\alpha \in M_{p}$, avec $f \neq 0$ :

$$
\begin{aligned}
A_{f}(h) & \leqslant A_{f}(g) A_{g}(h) \quad \text { si } g \neq 0 ; \\
A_{f}\left({ }_{\alpha} g\right) & \leqslant A_{f}(g) ; \\
A_{f}(g) & \geqslant\|g\| /\|f\| .
\end{aligned}
$$

De la relation $f={ }_{\delta} f$ et de (3), on déduit

$$
A_{f}(f)=1
$$

et on a évidemment

$$
A_{f}\left({ }_{\alpha} f\right) \leqslant 1
$$

puis (1) et (4) entraînent

$$
A_{f}(g) A_{g}(f) \geqslant 1 \quad \text { si } g \neq 0
$$

de (1) et (5), on déduit

$$
A_{f}(g) \leqslant A_{f}\left({ }_{\alpha} f\right) A_{a} f(g) \leqslant A_{a}(g) ;
$$

la relation suivante est évidente:

$$
A_{f}(g+h) \leqslant A_{f}(g)+A_{f}(h) .
$$

Si de plus $X$ est commutatif, on a

$$
A_{\alpha}\left({ }_{\alpha} g\right) \leqslant A_{f}(g)
$$

d'où les inégalités

$$
A_{f}\left({ }_{\alpha} g\right) \leqslant A_{\alpha} f\left({ }_{\alpha} g\right) \leqslant A_{f}(g) \leqslant A_{a}(g) .
$$

(La relation (9) est vérifiée lorsque $X$ est un groupe non commutatif, ce qui incite à la considérer comme plausible pour un hypergroupe quelconque.)

B. Choisissons maintenant, une fois pour toutes, une fonction $f_{0} \in K^{+}, f_{0}$ $\not \geq 0$, et posons, pour $f$ et $g \in L^{+}, f \neq 0, g \neq 0$,

$$
I_{f}(g)=A_{f}(g) / A_{f}\left(f_{0}\right) \text {. }
$$

On obtient alors, en vertu de (1), les inégalités

$$
0<a(g)=1 / A_{g}\left(f_{0}\right) \leqslant I_{f}(g) \leqslant A_{f_{0}}(g)=b(g) .
$$

Pour $g \equiv 0$, nous poserons par définition $a(g)=b(g)=0=I_{f}(g)$.

Pour tout voisinage $U$ de l'élément neutre $e$ de l'hypergroupe $X$, considérons une fonction $f_{U}$ bien déterminée, $\not \equiv 0$, appartenant à $K^{+}$et à support dans $U$. Pour $g . \in L^{+}$, écrivons $I_{U}(g)$ au lieu de $I_{f_{U}}(g)$. Nous normalisons $f_{U}$ par la condition $A_{f_{u}}\left(f_{0}\right)=1$.

Désignons par $\mathcal{Q}$ le filtre des voisinages de $e$, et par $A$ l'espace compact $\Pi_{g \in L^{+}}[a(g), b(g)]$; pour tout $U \in \mathcal{Q}$, soit $x_{U}$ le point de $A$ défini par $x_{U}=\left(I_{U}(g)\right)_{g \in L^{+}}$.

$A$ étant compact, il existe un point $x=(I(g))_{g \in L^{+}}$de $A$ tel que, pour tout 
$U \in \mathcal{Q}$ et tout voisinage $V$ de $x$ dans $A$, il existe $U^{\prime} \in \mathcal{Q}, U^{\prime} \subset U$ tel que $x_{U^{\prime}} \in V$. Evidemment, pour toute $g \in L^{+}, I(g) \in[a(g), b(g)]$.

C. Il est clair que, pour $g \in L^{+}$et $\alpha \in M_{p}, I\left({ }_{\alpha} g\right) \leqslant I(g)$, car pour tout $U \in \mathcal{Q}, I_{U}\left({ }_{\alpha} g\right)<I_{U}(g)$; on a évidemment $I(\lambda g)=\lambda I(g)$ pour $g \in L^{+}$et $\lambda>0$; montrons l'égalité $I(u+v)=I(u)+I(v)$ pour $u, v \in K^{+}$.

L'inégalité $I(u+v) \leqslant I(u)+I(v)$ résulte de $(8)$, pour $u$ et $v \in L^{+}$; vérifions l'inégalité inverse lorsque $u$ et $v$ sont de plus à support compact.

Soient d'abord $F, h, h^{\prime}$ trois fonctions de $K^{+}$, et $\varepsilon>0$. Appelons $S$ un compact contenant les supports de $h$ et de $h^{\prime}, T$ un voisinage compact de $S$. Il existe des ouverts relativement compacts $V_{j}$ et $W_{j}(1<j<n)$ tels que $\bar{W}_{j} \subset V_{j}$ pour tout $j$, que $T$ soit recouvert par les $W_{j}$ et que, sur chacun des $V_{j}$. l'oscillation de $h$ et de $h^{\prime}$ soit inférieure à $\varepsilon$. Il existe alors un voisinage $U$ de $e$ tel que le support de $\left(\delta_{y} * \delta_{x}\right)$ soit disjoint de $U$ quels que soient $x \in W_{j}, y \notin$ $V_{j}(1 \leqslant j \leqslant n)$.

Soient $f_{U}$ la fonction de $K^{+}(U)$ considérée précédement et $\mu \in M^{+}$une mesure telle que $F \leqslant{ }_{\mu} f_{U}$.

Alors, pour tout $x$ ( $j$ étant tel que $x \in W_{j}$ ),

$$
F(x) h(x) \leqslant h(x){ }_{\mu} f_{U}(x)=h(x) \int\left\langle\delta_{y} * \delta_{x}, f_{U}\right\rangle d \mu(y) ;
$$

or d'après le choix de $U,\left\langle\delta_{y} * \delta_{x}, f_{U}\right\rangle$ est nul si $y \notin V_{j}$, donc

$$
F(x) h(x) \leqslant \int(h(y)+\varepsilon)\left\langle\delta_{y} * \delta_{x}, f_{U}\right\rangle d \mu(y)={ }_{(h+\varepsilon) \mu} f_{U^{\prime}}(x),
$$

et

Par conséquent

$$
I_{U}(F h) \leqslant \int(h+\varepsilon) d \mu .
$$

$$
I_{U}(F h)+I_{U}\left(F h^{\prime}\right) \leqslant \int\left(h+h^{\prime}+2 \varepsilon\right) d \mu .
$$

Si $h+h^{\prime}<1$, et si on chosit $\mu$, ce qui possible, telle que $\|\mu\|$ soit suffisamment proche de $I_{U}(F)$, on en déduit $I_{U}(F h)+I_{U}\left(F h^{\prime}\right) \leqslant(1+3 \varepsilon) I_{U}(F)$ et, compte tenu de la définition de $I$,

$$
I(F h)+I\left(F h^{\prime}\right) \leqslant I(F) .
$$

Revenons maintenant aux fonctions de $K^{+}, u$ et $v$, données. Soit $p \in K^{+}$ une fonction $>0$ sur le support de $u+v$ et $\varepsilon$ un nombre $>0$. Posons $F=u+v+\varepsilon p, h=u / F, h^{\prime}=v / F$. Nous sommes bien dans les conditions précédentes, ce qui permet d'écrire

$$
I(u)+I(v)<I(u+v+\varepsilon p)<I(u+v)+\varepsilon I(p)
$$

d'où le résultat en faisant tendre $\varepsilon$ vers 0 .

Ceci prouve, compte tenu des autres propriétés, que $I$, après prolongement linéaire à $K$, est une mesure de Radon. Notons $m$ cette mesure. 
Remarquons que nous avons obtenu une mesure dont le support soit $X$ entier (résultat annoncé antérieurement).

D. Si Maintenant $g$ est une fonction de $L^{+}$qui n'appartient pas à $K^{+}, g$ est $m$-intégrable et $m(g) \leqslant I(g)$, car $m(g)$ est la borne supérieure des nombres $m(f)=I(f) \leqslant I(g)$, pour les $f \in K^{+}, f \leqslant g$; d'autre part, si $g$ et $h \in L^{+}$, on n'a pas établi l'égalité $I(g+h)=I(g)+I(h)$; par contre, bien entendu, $m(g+h)=m(g)+m(h)$. Pour $g \in K^{+}$et $\alpha \in M_{p}, m\left({ }_{\alpha} g\right) \leqslant m(g)$.

Jusqu'ici, $X$ n'a pas été supposé commutatif (sauf pour la relation (9) qui n'a pas encore été utilisée). Il le sera désormais.

LEMME III.1. Soient $X$ un hypergroupe commutatif, $g$ et $h$ deux éléments de $L^{+}, \not \equiv 0$; on $a$

$$
1 / A_{g}(h) \leqslant m(g) / m(h) \leqslant A_{h}(g) .
$$

Remarquons que les inégalités

$$
1 / A_{g}(h) \leqslant I(g) / I(h) \leqslant A_{h}(g)
$$

sont immédiates, compte tenu de ce qui précède. Mais la situation se complique du fait que nous ignorons si $m$ et $I$ coincident sur $L^{+}$.

Soit donc $\mu \in M^{+}$telle que $g \leqslant_{\mu} h$, et soit $\left\{h_{k}\right\}_{k>0}$ une suite de $K^{+}$qui converge vers $h$ en croissant (une telle suite existe toujours, car $h$, appartenant à $L^{+}$, est nulle hors d'un ensemble $\sigma$-compact, propriété qui se déduit aisément du Lemme II.9) alors la suite $\left\{{ }_{\mu} h_{k}\right\}_{k>0}$ converge en croissant vers ${ }_{\mu} h$ (théorème de convergence monotone); la convergence est même uniforme sur tout compact d'après le lemme de Dini. Soit $g^{\prime} \in K^{+}$, telle que, sur son support, $g^{\prime}$ soit strictement inférieure à $g$; alors $g^{\prime}$ est majorée par ${ }_{\mu} h_{k}$ pour tout $k$ assez grand, de sorte que (pour $k$ assez grand)

$$
m\left(g^{\prime}\right) / m\left(h_{k}\right)=I\left(g^{\prime}\right) / I\left(h_{k}\right) \leqslant A_{h_{k}}\left(g^{\prime}\right) \leqslant\|\mu\| ;
$$

comme $m(g)=$ sup $m\left(g^{\prime}\right)$, la borne supérieure pouvant être prise dans l'ensemble des $g^{\prime} \in K^{+}$assujetties aux conditions ci-dessus, on obtient, en faisant tendre $k$ vers l'infini,

$$
m(g) / m(h) \leqslant\|\mu\|
$$

d'où

$$
m(g) / m(h) \leqslant A_{h}(g) .
$$

L'autre inégalité s'obtient en échangeant $g$ et $h$.

E. Considérons maintenant, $X$ étant un hypergroupe commutatif, l'espace vectoriel $E$ des mesures de Radon sur $X$ par rapport auxquelles toutes les fonctions de $L$ sont intégrables. Munissons $E$ de la topologie faible $\sigma(E, L)$.

Lemme III.2. Soient $X$ un hypergroupe commutatif et $f_{0}$ une fonction de $K^{+}, \not \equiv$, choisie une fois pour toutes. Pour toute fonction $g \in L^{+}$, posons 
$a(g)=1 / A_{g}\left(f_{0}\right), b(g)=A_{f_{0}}(g)$ si $g \not \equiv 0$ (alors $\left.0<a(g) \leqslant b(g)\right)$, et $a(g)=$ $b(g)=0$ si $g \equiv 0$.

$L$ 'ensemble $A$ des mesures positives $m$ appartenant à $E$, ayant $X$ pour support, telles que $m\left(f_{0}\right)=1$ et que, pour toute $g \in L^{+}$et toute $\alpha \in M_{p}$, l'on ait

$$
a(g) \leqslant m\left({ }_{\alpha} g\right) / m\left({ }_{\alpha} f_{0}\right) \leqslant b(g),
$$

est une partie non vide, convexe et compacte de $E$, ne contenant pas la mesure 0 .

D'abord, la mesure particulière $m$ construite plus haut satisfait, d'après le Lemme III.1 et la relation (9) valable lorsque $X$ est commutatif, aux inégalités:

$$
\begin{gathered}
a(g)=1 / A_{g}\left(f_{0}\right) \leqslant 1 / A_{\alpha g}\left({ }_{\alpha} f_{0}\right) \leqslant m\left({ }_{\alpha} g\right) / m\left({ }_{\alpha} f_{0}\right) \\
A_{\alpha} f_{0}\left({ }_{\alpha} g\right) \leqslant A_{f_{0}}(g)=b(g)
\end{gathered}
$$

si $g \neq 0$ (relations évidentes si $g \equiv 0$ ), donc appartient à l'ensemble $A$ (après normalisation par la condition $\left.m\left(f_{0}\right)=1\right)$, donc $A \neq \varnothing$. La convexité de $A$ est immédiate. D'autre part, $A$ est évidemment fermé dans $E$ et borné, donc compact. De plus la mesure nulle ne saurait appartenir à $A$.

Pour toute mesure $m$ de $A$ et toute $\alpha \in M_{p}$, soit $T_{\alpha}(m)$ la mesure, appartenant à $E$, définie par $T_{\alpha}(m)(g)=m\left({ }_{\alpha} g\right) / m\left({ }_{\alpha} f_{0}\right)$ pour $g \in K^{+}$. Il est clair que $T_{\alpha}$ applique $A$ dans $A$, de manière continue. Mais $T_{\alpha}$ n'est pas une application affine de $A$ dans $A$.

La famille des transformations $T_{\alpha}$ est commutative (si $X$ est un hypergroupe commutatif). Si les $T_{\alpha}$ étaient affines, le theorème du point fixe de Markov-Kakutani prouverait l'existence d'une mesure $m$ telle que $T_{\alpha}(m)$ $=m$ pour toute $\alpha \in M_{p}$ : une telle mesure serait relativement invariante.

Mais les $T_{\alpha}$ ne sont pas affines: elles ont une propriété plus faible, qui est de transformer tout intervalle d'extrémités $m_{1}, m_{2}$ dans $A$ en l'intervalle d'extrémités $T_{\alpha}\left(m_{1}\right), T_{\alpha}\left(m_{2}\right)$. De même, dualement, l'application qui, pour une mesure $m$ fixée de $A$, associe à $\alpha \in M_{p}$ la mesure $T_{\alpha}(m)$, n'est pas affine, mais a la propriété analogue de transformer l'intervalle d'extrémités $\alpha, \beta$ dans $M_{p}$ en l'intervalle d'extrémités $T_{\alpha}(m), T_{\beta}(m)$ dans $A$. C'est cette dernière propriété qui va nous permettre de conclure.

F. Il nous faut d'abord établir un résultat intéressant pour lui-même.

THEOREME III.3. Soit $A$ une partie compacte convexe non vide d'un espace vectoriel topologique localement convexe. Soit $\mathcal{C}$ une famille commutative d'applications continues de $A$ dans $A$, ayant les deux propriétés suivantes:

(a) $\mathcal{C}$ est stable par composition;

(b) $\mathcal{C}$ est convexe, c'est-à-dire: pour tout $x \in A$, l'ensemble $\{T(x), T \in \mathfrak{E}\}$ est une partie convexe de $A$.

Alors il existe dans $A$ un point invariant par chacune des applications de la famille $\mathrm{C}$. 
Ce résultat peut-être considéré comme une généralisation des deux théorèmes classiques de point fixe, de Markov-Kakutani et de SchauderTychonoff $\left({ }^{2}\right)$.

Le théorème de Schauder-Tychonoff affirme que chacune des applications de la famille $\mathcal{C}$ a des points fixes. Pour tout $T \in \mathcal{E}$, soit $F(T)$ l'ensemble non vide des points fixes de $T$. Il s'agit de montrer que l'intersection des $F(T)$ n'est pas vide. Comme chacun des ensembles $F(T)$ est une partie fermée du compact $A$, il suffit de montrer qu'une intersection finie d'ensembles $F(T)$ n'est pas vide.

Il suffit donc de montrer ceci: soient $T_{1}, \ldots, T_{n}, T_{0}, n+1$ applications appartenant à $\mathcal{C}$ et $F_{1}, \ldots, F_{n}, F_{0}$ leurs ensembles respectifs de points fixes; si $F_{1} \cap \cdots \cap F_{n} \cap F_{0} \neq \varnothing$, alors $F_{1} \cap \cdots \cap F_{n} \cap F_{0} \neq \varnothing$.

Faisons donc l'hypothèse $F_{1} \cap \cdots \cap F_{n} \neq \varnothing$. Soit $x$ un point appartenant à cette intersection; l'ensemble $B=\left\{T(x), T \in \mathcal{C}^{\prime}\right\}$ est convexe par hypothèse et contenu dans $F_{1} \cap \cdots \cap F_{n}$ puisque la famille $E$ est commutative; de plus, $T_{0}(B) \subset B$ puisque $\mathcal{C}$ est stable par composition; si $C$ désigne l'adhérence de $B$ dans $A, C$ est convexe compacte et $T_{0}(C) \subset C$ par continuité; le théorème de Schauder-Tychonoff s'applique alors à la restriction de $T_{0}$ à $C$, d'où un point fixe de $T_{0}$ dans $C$; comme $C$ est contenu dans $F_{1} \cap \cdots \cap F_{n}$, le théorème est ainsi démontré.

G. Revenons à la situation antérieure, où $A$ est la partie convexe compacte non vide définie au Lemme III.2 de l'espace localement convexe $E$.

Nous appliquerons le Théorème III. 3 en prenant pour $\bigodot$ la famille $\left\{T_{\alpha}, \alpha\right.$ $\left.\in M_{p}\right\}$. Les deux conditions du théorème sont satisfaites: $巳$ est bien stable par composition, puisque $T_{\alpha} T_{\beta}=T_{\alpha * \beta}$; vérifions la condition de convexité: soient $m \in A$ et $\alpha$ et $\beta \in M_{p}, 0 \leqslant t \leqslant 1$; il s'agit de montrer qu'il existe $\gamma \in M_{p}$ telle que $t T_{\alpha}(m)+(1-t) T_{\beta}(m)=T_{\gamma}(m)$. Or, pour toute $g \in L^{+}$,

$$
\left\{t T_{\alpha}(m)+(1-t) T_{\beta}(m)\right\}(g)=t \frac{m\left({ }_{\alpha} g\right)}{m\left({ }_{\alpha} f_{0}\right)}+(1-t) \frac{m\left({ }_{\beta} g\right)}{m\left({ }_{\beta} f_{0}\right)},
$$

et ceci est bien de la forme $T_{\gamma}(m)(g)=m\left({ }_{\gamma} g\right) / m\left({ }_{\gamma} f_{0}\right)$ si la mesure $\gamma$ est définie par $\gamma=u \alpha+(1-u) \beta$ avec

$$
u=\frac{t / m\left({ }_{\alpha} f_{0}\right)}{t / m\left({ }_{\alpha} f_{0}\right)+(1-t) / m\left({ }_{\beta} f_{0}\right)} .
$$

La démonstration est maintenant achevée: il existe une mesure $m \in A$, invariante pour toute transformation $T_{\alpha}$ : c'est-à-dire que $\forall \alpha \in M_{p}$ et $\forall g \in$ $L, T_{\alpha}(m)(g)=m\left({ }_{\alpha} g\right) / m\left({ }_{a} f_{0}\right)=m(g)$, et ceci prouve que $m$ est relativement invariante; d'où le théorème:

(2)Voir par exemple Dunford-Schwartz, Linear operators, t. 1. 
THEOREME III.4. Tout hypergroupe commutatif possède une mesure invariante.

Ceci s'obtient à partir de ce qui précède, en appliquant le Théorème II.4.

\section{REFERENCES}

1. Ch. F. Dunkl, The measure algebra of a locally compact hypergroup, Trans. Amer. Math. Soc. 179 (1973), 331-348. MR 47 \#9171.

2. R. I. Jewett, Spaces with an abstract convolution of measures, Advances in Math. 18 (1975), 1-101. MR 52 \# 14840.

3. K. A. Ross, Hypergroups and centers of measure algebras, A paraitre dans Symposia Mathematica, Istituto Nazionale di alta Matematica.

4. R. Spector, Théorie axiomatique des hypergroupes, C. R. Acad. Sci. Paris Sér. A 280 (1975), 1743-1744. MR 52 \#11472 A.

5. __ Apercu de la théorie des hypergroupes, Analyse Harmonique sur les Groupes de Lie, Séminaire Nancy-Strasbourg 1973-1975, Lecture Notes in Math. vol. 497, Springer-Verlag, Berlin and New York. 1975.

6. A. Weil, L'intégration dans les groupes topologiques et ses applications, Hermann, Paris, 1940. MR 3, 198.

Département de Mathématiques, Université d'Orléans, 45045 Orléans, France 